\title{
Schöne neue Welt
}

Viele Unternehmen stellen ihren Mitarbeitern anstelle von stationären Arbeitsplatzrechnern mobile Notebooks zur Verfügung. Technisch unterscheiden diese Geräte sich kaum von ihren stationären Pendants. Betriebssystem und verbaute Hardware sind weitgehend identisch. Bedienung und Nutzverhalten weichen kaum von stationären Arbeitsplatzrechnern ab. Das mobile Arbeiten wird meist durch besonders gesicherte Datenkanäle oder eine virtualisierte Arbeitsumgebung realisiert.

Bei Smartphones und Tablet-Computern ist dies anders. Diese Geräte sind für den Consumer-Markt entwickelt. Hardware und Systemsoftware sind auf die privaten Belange ihrer Nutzer abgestimmt. Ihr Bedienkonzept und die jederzeit durch die Installation von Apps erweiterbaren Funktionalitäten haben zu einem neuen Nutzerverhalten geführt. Während Notebooks im Privatbereich zur Verwaltung von medialen Inhalten wie Musik, Fotos oder Videos verwendet werden, haben sich Smartphones zur mobilen Allzweckwaffe entwickelt. Im Privatbereich erfolgt die Kommunikation kaum noch via E-Mail. Nachrichten, Fotos und andere mediale Inhalte werden heute über WhatsApp, Facebook, Twitter, Skype und Co. ausgetauscht. Durch die ständige Verbindung mit dem Internet und entsprechende Apps kann über das Smartphone überall und jederzeit auf Inhalte zugegriffen werden. Smartphones sind mangels eines zeitraubenden Boot-Vorgangs sofort einsatzbereit.

All dies hat dazu geführt, dass Arbeitnehmer die ihnen aus dem Privatbereich bekannten Annehmlichkeiten auch im beruflichen Umfeld nutzen wollen.

T. Walter, Bring your own Device - Ein Praxisratgeber, essentials, DOI 10.1007/978-3-658-11591-3_1 